\title{
Effect of treatment with the antioxidant alpha-lipoic (thioctic) acid on heart and kidney microvasculature in spontaneously hypertensive rats
}

\author{
Seyed Khosrow Tayebati ${ }^{1}$, Daniele Tomassoni ${ }^{2}$, Lorenzo Di Cesare Mannelli ${ }^{3}$, and Francesco Amenta ${ }^{1}$ \\ ${ }^{1}$ School of Pharmacy, University of Camerino, Camerino, Italy, ${ }^{2}$ School of Bioscience and Veterinary Medicine, University of Camerino, Camerino, \\ Italy, and ${ }^{3}$ Department of Clinical and Preclinical Pharmacology, University of Florence, Florence, Italy
}

\begin{abstract}
Endothelial cells represent an important vascular site of signaling and development of damage during ischemia, inflammation and other pathological conditions. Excessive reactive oxygen species production causes pathological activation of endothelium including exposure of cell to adhesion molecules. Intercellular adhesion molecule-1 (ICAM-1), vascular cell adhesion molecule-1 (VCAM-1) and platelet-endothelial cell adhesion molecule-1 (PECAM-1) are members of the immunoglobulin super-family which are present on the surface of endothelial cells. These molecules represent important markers of endothelial inflammation. The present study was designed to investigate, with immunochemical and immunohistochemical techniques, the effect of treatment with (+/-)-alpha lipoic (thioctic) acid and its enantiomers on heart and kidney endothelium in spontaneously hypertensive rats (SHR). Arterial hypertension is accompanied by an increased oxidative stress status in the heart characterized by thiobarbituric acid reactive substances (TBARS) and nucleic acid oxidation increase. The higher oxidative stress also modifies adhesion molecules expression. In the heart VCAM-1, which was higher than ICAM-1 and PECAM-1, was increased in SHR. ICAM-1, VCAM-1 and PECAM-1 expression was significantly greater in the renal endothelium of SHR. (+/-)-Alpha lipoic acid and (+)-alpha lipoic acid treatment significantly decreased TBARS levels, the nucleic acid oxidation and prevented adhesion molecules expression in cardiac and renal vascular endothelium. These data suggest that endothelial molecules may be used for studying the mechanisms of vascular injury on target organs of hypertension. The effects observed after treatment with (+)-alpha lipoic acid could open new perspectives for countering heart and kidney microvascular injury which represent a common feature in hypertensive end-organs damage.
\end{abstract}

\author{
Keywords \\ Alpha-lipoic acid, endothelial adhesion \\ molecules, spontaneously hypertensive \\ rats
}

\section{History}

Received 19 March 2015

Revised 24 April 2015

Accepted 25 April 2015

Published online 24 July 2015

\section{Introduction}

Arterial hypertension represents the main risk factor for morbidity and mortality associated with cardiovascular diseases. Heart and kidney are main target organs of hypertension (1-3). Other obvious target organs of hypertension are arteries and arterioles. Hypertension affects the arterial walls leading to an increase (hypertrophy) of wall size and/or vascular tone accompanied by reduction of the vascular lumen $(4,5)$. This phenomenon increases peripheral resistance that may be complicated by a vascular stenosis with reduced organ perfusion (6). Accumulation of intimal vascular smooth muscle cells is a trait of atherosclerosis. Endothelial cells lining vascular luminal surface represent an important site of signaling and development of damage induced by reactive oxygen species (ROS) during ischemia, inflammation and other pathological conditions (6).

Targeted delivery of ROS modulating enzymes conjugated with antibodies to endothelial surface molecules provides

Correspondence: Seyed Khosrow Tayebati, PharmD, PhD, School of Pharmacy, University of Camerino, Via Madonna delle Carceri, 9, 62032 Camerino (MC), Italy. Tel: +390737403305. Fax: +390737403325. E-mail: khosrow.tayebati@unicam.it site-specific effects leading to endothelial damage. Excessive ROS production induces pathological activation of endothelium including exposure of cell adhesion molecules. The intercellular adhesion molecule-1 (ICAM-1) is a member of the immunoglobulin $(\mathrm{Ig})$ superfamily which is present at the surface of several cell types, including endothelial cells. ICAM-1 and other adhesion molecules such as vascular cell adhesion molecule 1 (VCAM-1), platelet-endothelial cell adhesion molecule-1 (PECAM-1), expressed on activated endothelium, could represent a pharmacological target and specific sites for imaging probe for evaluating vascular pathologies. (7-9).

Alpha-lipoic acid, (1,2-dithiolane-3-pentanoic acid), or thioctic acid is a naturally occurring dithiol compound synthesized enzymatically in the mitochondria from octanoic acid. It is synthesized in the organism and is absorbed intact from dietary sources. After absorption, it accumulates transiently in many tissues. There is growing evidence that orally delivered alpha-lipoic acid may not be used as a metabolic cofactor but instead, it elicits a unique set of biochemical activities with potential therapeutic value against different pathophysiologic insults. Due to the presence of an asymmetric carbon $\mathrm{C} 3$, alpha-lipoic acid exists in two enantiomers, 
namely (+)- and (-)-alpha-lipoic acid. The former enantiomer represents the active form of the compound. It is located intracellularly and elicits the biological effects of alpha-lipoic acid (10). Moreover, (-)-alpha-lipoic acid acts either as a poor substrate or as an inhibitor of (+)-alpha-lipoic acid when it interacts with 2-oxoacid dehydrogenase multienzyme complexes.

The antioxidant activity of alpha-lipoic was investigated in an animal model of hypertension such as spontaneously hypertensive rats (SHR) $(11,12)$. SHR has specific and uniform genetic predisposition to develop arterial hypertension (13), allowing to study causes, mechanisms, pathology and behavioral consequences of the disease. Arterial hypertension also represents an important cause of oxidative stress. In fact, it could be considered as a free radical generating source (14) and, therefore, SHR can be used as an animal model of oxidative stress. Hypertension-dependent organ damage was demonstrated (13,15-17). These studies have shown that different organs of SHR (e.g., kidney, heart and brain), undergo to hypertension-related ROS-depended damage (18). Hence, SHR may be a useful animal model to study the effect of any antioxidant compound.

Therefore, the present study was designed to investigate with immunochemical and immunohistochemical techniques, the effect of treatment with alpha-lipoic acid enantiomers on heart and kidney endothelium in SHR used as a model of hypertensive end organ damage where vascular tree develops of smooth muscle layer thickness and lumen narrowing.

\section{Materials and methods}

\section{Animals and tissue treatment}

Twenty-week-old male SHR $(n=30)$ and age-matched WKY rats $(n=6)$ were used. Rats were fed with standard laboratory diet and with tap water ad libitum, and kept at $23 \pm 1{ }^{\circ} \mathrm{C}$ with a $12 \mathrm{~h} \mathrm{light/dark} \mathrm{cycle,} \mathrm{light} \mathrm{at} 7$ a.m. Animal manipulation was carried out according to the National and European Community Guidelines for Animal Care (DL 116/92, of application of the European Communities Council Directive 86/609/EEC) and also in accordance with the ethical guidelines of the University of Florence. The guidelines deal with treatment of animals under lab testing. These guidelines are consistent with the Guide for the Care and Use of Laboratory Animals of the US National Institutes of Health (NIH Publication No. 85-23, revised 1996; University of Florence assurance number: A5278-01). All efforts were made to minimize animal suffering and to reduce the number of animals used.

SHR rats were treated once a day for 30 days with an i.p. injection of $250 \mu \mathrm{mol} / \mathrm{kg} /$ day of $(+/-)$-alpha-lipoic acid $(n=6) ; 125 \mu \mathrm{mol} / \mathrm{kg} /$ day of $(+/-)$-alpha-lipoic acid $(n=6)$; $125 \mu \mathrm{mol} / \mathrm{kg} /$ day of $(+)$-alpha-lipoic acid lysine salt $(n=6)$ and $125 \mu \mathrm{mol} / \mathrm{kg} /$ day of $(-)$-alpha-lipoic acid $(n=6)$. WKY $(n=6)$ and SHR $(n=6)$ rats were also treated with the same amounts of vehicle as control.

Animals were anaesthetised with pentobarbital sodium $(50 \mathrm{mg} / \mathrm{kg}$, by intraperitoneal route), and then perfused through the left ventricle with a $0.9 \% \mathrm{NaCl}$ solution containing $0.5 \%$ polyvinylpyrrolidone, heparin (20 international units), and ethylene diamine tetra-acetic acid
(EDTA, $25 \mathrm{mg} / \mathrm{ml}$ ) to induce a maximal vasodilatation. The first solution was then replaced by a second solution of $10 \%$ formalin in $0.1 \mathrm{M}$ phosphate buffer ( $\mathrm{pH} 7.4)$, kept at room temperature. After perfusion, kidney and heart were dissected out, weighed and fixed in the fixative and embedded into paraffin wax. Serial consecutive 12 or $8 \mu \mathrm{m}$ thick sections were cut using a rotary microtome and processed for morphological analysis and immunohistochemistry as detailed below.

\section{Thiobarbituric acid reactive substances (TBARS) determination}

Samples $(0.1 \pm 0.02 \mathrm{~g})$ of heart and kidney were homogenized in a Mixer Mill MM300 (Qiagen, Hilden, Germany) in specific lysis buffer. After homogenization, the samples were centrifuged (2 times) at $13000 \mathrm{rpm}\left(10 \mathrm{~min}\right.$ at $\left.4{ }^{\circ} \mathrm{C}\right)$. Aliquots of the supernatant were used for protein assay against a standard of bovine serum albumin (BSA) using a BIO-RAD protein assay (BIO-RAD, Munich, Germany). Levels of thiobarbituric acid reactive substances (TBARS) were measured using commercial kits (Cat. No. 10009055, Cayman Chemical Company, Cayman, Ann Arbor, MI, USA) and the value expressed as $\mathrm{pmol} / \mathrm{mg}$ of tissue.

\section{Western blot analysis}

Equal amount of proteins $(40 \mu \mathrm{g})$, obtained using the same procedures quoted in the last paragraph, was separated by $8 \%$ sodium dodecyl sulfate polyacrylamide gel electrophoresis. Transblotted membranes were incubated with different polyclonal antibodies (ICAM-1, Sc-8439; VCAM-1, Sc-8304; and PECAM-1, Sc-1506) that were purchased from Santa Cruz Biotechnology, Inc, Dallas, TX, USA, and were used at 1:500 dilution.

Immunochemistry product was visualized using as HRPbiotinylated antibody (donkey anti-goat IgG cat. No. Sc-2020, donkey anti-rabbit IgG cat. No. Sc-2313 or donkey antimouse cat. No. Sc 2314, Santa Cruz Biotechnology, Inc, Dallas, TX, USA) followed by a chemiluminescence detection system (Lite Ablot ${ }^{\circledR}$ plus, Cat. EMP 011005, Euroclone, Pero, Italy) with computer-driven densitometry. To normalize protein loadings, membranes were stripped and incubated with a monoclonal anti-GAPDH antibody (Cat. no 9295 Sigma Chemical Co., St. Louis, MO, USA) or anti beta-actin (clone AC-74; Sigma Chemical Co., St. Louis, MO, USA). Band intensities were measured by densitometry with an IAS 2000 image analyzer (Biosystem, Rome Italy). Densitometry values for each band were normalized to the respective reference protein intensity for each sample and referred to the normalized value of WKY group for different endothelial markers.

\section{Vascular morphometry by image analysis}

The influence of hypertension and antioxidant treatment on the coronary and renal arteries was assessed by image analysis. Alternate consecutive sections $(6 \mu \mathrm{m}$ thick, $50 \mu \mathrm{m}$ apart) were stained with Masson's trichromic protocol used to assess the morphology of different heart parenchymal and 
vascular components. Slides stained with Masson's trichrome were viewed at a microscope connected by a video camera to an image analyzer (IAS 2000, Delta Sistemi, Rome, Italy). Coronary and renal arteries were divided based on their external diameter range, as previously described $(19,20)$.

\section{Immunohistochemistry}

Consecutive sections ( $12 \mu \mathrm{m}$ thick) were processed for the immunohistochemical detection of 8-Oxo-2'-deoxyguanosine (8-oxo-dG) to analyze the DNA oxidative status and endothelial markers ICAM-1, VCAM-1, PECAM-1.

The sections were processed for the immunoistochemical detection of 8-oxo-dG using the polyclonal antibody (clone 2E2, Trevigen, cat.No. 4354-MC-050) diluted 1:250 in PBS containing $0.1 \%$ of BSA. In accordance with the company protocol, the sections were pre-treated at $37^{\circ} \mathrm{C}$ with $5 \mu \mathrm{g} / \mathrm{ml}$ Proteinase $\mathrm{k}$ in phosphate buffer saline (PBS) for $30 \mathrm{~min}$ and $100 \mu \mathrm{g} / \mathrm{ml}$ RNase A in $15 \mathrm{mM}$ sodium citrate buffer containing $150 \mathrm{mM} \mathrm{NaCl}$ for $60 \mathrm{~min}$. For the immunohistochemical detection of endothelial markers, the sections were incubated with the same antibodies, used for western blot analysis, at 1:100 dilution.

After a pre-incubation in 3\% PBS-bovine serum albumin for $1 \mathrm{~h}$, the sections were exposed overnight in a moist chamber at $4{ }^{\circ} \mathrm{C}$ to primary antibodies and then for $30 \mathrm{~min}$ at $25^{\circ} \mathrm{C}$ to corresponding secondary biotinylated antibodies (goat antirabbit IgGs, cat. No. 16-15-06 KPL, USA; rabbit anti-goat IgGs, cat.No. 16-13-06 KPL, USA; goat anti-mouse IgGs, AP124B, Merck Millipore, Life Science of Merck KGaA, Darmstadt, Germany) diluted to 1:200. The product of immune reaction was revealed using 3,3'-diaminobenzidine as a chromogen. A section of each series were used as a negative control and exposed to a non-immune IgG instead of the primary antibody. The density of immunoreactions was detected by image analysis system using overlapping function to define the endothelial layer as described elsewhere (21). The intensity was expressed as arbitrary optical density unit. Measurements were made in blind by two operators independently.

\section{Data analysis}

The averages of different parameters investigated were calculated from single animal data, and group means \pm S.E.M. were then derived from mean single animal values. The significance of the differences between the averages was analyzed by analysis of variance (ANOVA) followed by the Newman-Keuls multiple range test. Differentsized artery branches data were also grouped per vessel external diameter. Morphometric values were referred to diameter classes per similar size arteries grouped according to lumen size distribution curve (22).

\section{Results}

Body weight values were similar in normotensive WKY rats and SHR, either control or treated with different formulations of alpha-lipoic acid (data not shown). No significant differences were found between WKY and SHR or SHR treated with alpha-lipoic acid (data not shown). Heart weight/ body weight ratio was increased in control SHR rats $(3.37 \pm 0.12)$ compared to control WKY rats $(3.05 \pm 0.06)$. This phenomenon was not countered by antioxidant treatment (data not shown). Neither hypertension nor pharmacologic treatments changed kidney weight values (data not shown). Systolic blood pressure values were higher in control SHR rats $(215 \pm 3 \mathrm{mmHg})$ compared to the normotensive WKY rats $(148 \pm 9 \mathrm{mmHg})$. Treatment with any stereoisomer of alpha-lipoic acid did not significantly affect blood pressure values in SHR (data not shown).

\section{TBARS levels and 8-oxo-dG immunohistochemistry}

In SHR an increased oxidative stress characterized by a significant rise of TBARS level in heart (Figure 1A) was observed compared to WKY rats. Treatment with (+)-thioctic acid $(125 \mu \mathrm{mol} / \mathrm{kg} / \mathrm{day})$ significantly decreased TBARS (Figure 1A). The two different doses of (+/-)-thioctic acid had only a slight effect on TBARS (Figure 1A). In the kidney of SHR no significant increase of TBARS level was detected compared to WKY rats (Figure 1B). Treatment with (+/-)-thioctic acid $(250 \mu \mathrm{mol} / \mathrm{kg} / \mathrm{day})$ and with (+)-thioctic acid $(125 \mu \mathrm{mol} / \mathrm{kg} /$ day) significantly decreased TBARS level (Figure 1B).

8-Oxo-dG immunostaining was expressed in a thin granular staining localized in the nuclei of the smooth muscle cells of the heart and kidney arteries (Figure 2). Immunoreaction was more pronounced in SHR compared to WKY (Figure 2). Treatment with the (+)-thioctic acid decreased the 8-oxo-dG immunoreaction (Figure 2).

\section{Vascular morphometry}

Analysis of different-size coronary arteries with $100-50 \mu \mathrm{m}$ of diameter of control SHR revealed an increase of wall area $\left(2723.95 \pm 148.06 \mu \mathrm{m}^{2}\right)$ and luminal narrowing (830.01 \pm $37.75 \mu \mathrm{m}^{2}$ ) compared to size-matched arteries of WKY rats wall area $\left(2386.25 \pm 221.70 \mu \mathrm{m}^{2}, p<0.05\right.$ vs SHR) and lumen area $\left(1057.23 \pm 93.60 \mu \mathrm{m}^{2}, p<0.05\right.$ vs SHR). An increase of the wall-to-lumen ratio was also noticeable $(3.30 \pm 0.14$ for SHR and $2.25 \pm 0.19$ for WKY, $p<0.05$ vs SHR). In differentsized coronary arteries, treatment with the racemic formulation at the two concentrations and the enantiomers has shown no effect on wall area and luminal narrowing (data not shown). No remodeling occurred in different-sized coronary artery branches of control or antioxidant-treated SHRs compared with normotensive WKY rats (data not shown).

Renal arteries with $100-50 \mu \mathrm{m}$ of diameter of control SHRs compared with size-matched arteries of WKY shows the increased thickness of the tunica media $(3092.70 \pm 201.30$ SHR and 2814.21 \pm 219.11 for WKY, $p<0.05$ vs SHR) accompanied by luminal narrowing $(709.63 \pm 50.94$ SHR and $1044.53 \pm 75.17$ for WKY, $p<0.05$ vs SHR). A consequent significant augmentation of the wall-to-lumen ratio was also found $(4.52 \pm 0.34$ for SHR and $3.89 \pm 0.54$ for WKY, $p<0.05$ vs SHR). An accumulation of connective tissue was also observed in the tunica adventitia of different sized of renal arteries of SHRs (data not shown). Enantiomers of alpha-lipoic acid and the racemic formulation at two concentrations had no effect on the vascular changes due to hypertension (data not shown). 

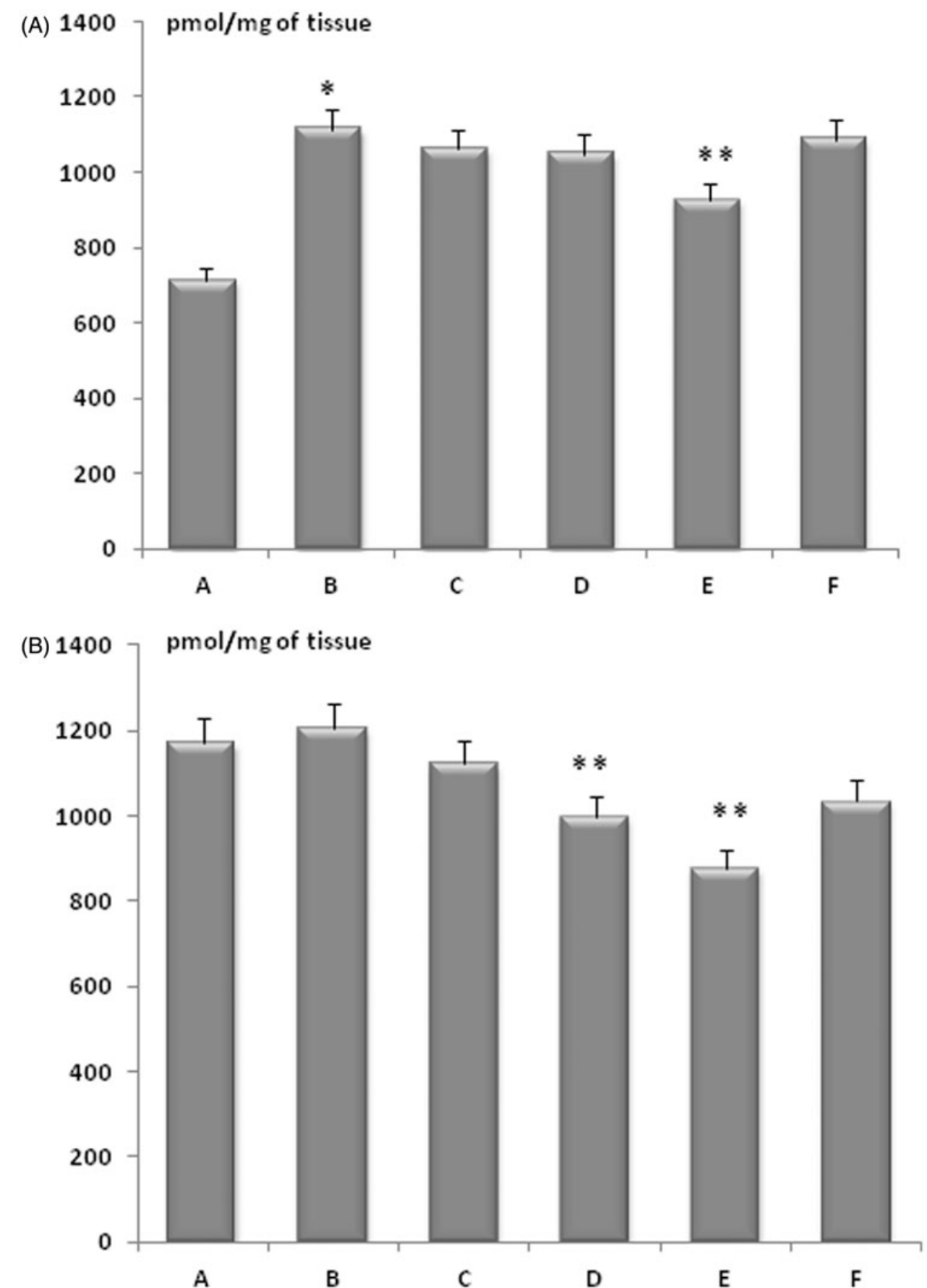

Figure 1. TBARS levels in heart (A) and kidney (B) tissue homogenates, expressed as pmol/mg of tissue. A: WKY + FIS B: SHR + FIS C: SHR + (+/-)-alpha lipoic acid $125 \mu \mathrm{mol} / \mathrm{kg} / \mathrm{day}$; D:SHR CCI + (+/-)alpha lipoic acid $250 \mu \mathrm{mol} / \mathrm{kg} / \mathrm{day} ; \mathrm{E}: \mathrm{SHR}+(+)$ alpha lipoic acid $125 \mu \mathrm{mol} / \mathrm{kg} / \mathrm{day} ; \mathrm{F}$ : SHR + (-)alpha lipoic acid $125 \mu \mathrm{mol} / \mathrm{kg} /$ day. $* p<0,05$ versus WKY + FIS rats; $* * p<0.05$ versus SHR + FIS rats.

\section{Immunochemical and immunohistochemical analysis}

Immunochemical analysis was performed on samples of heart and kidney of animals treated and untreated with different doses of $(+/-)$-alpha-lipoic and its enantiomers for the evaluation of the expression of adhesion factors ICAM-1, VCAM-1 and PECAM-1. The analysis revealed a band approximately $90 \mathrm{kDa}$ for ICAM-1, $100 \mathrm{kDa}$ for VCAM-1 and $120 \mathrm{kDa}$ for PECAM-1 approximately (Figure 3). Evaluation of the different bands was made for both the heart and the kidney referring to density of GAPDH or $\beta$-actin reference proteins. The expression of adhesion molecules was significantly increased in heart and kidney of SHR (Figure 3).
Treatment with alpha-lipoic acid enantiomer or racemic formulations partially prevents the damage caused by hypertension. In fact, the (+)-alpha-lipoic acid $125 \mu \mathrm{mol} / \mathrm{kg} /$ day is the more affective enantiomer to counter VCAM-1 expression in cardiac and renal homogenates (Figure 3). ICAM-1and PECAM-1 expression was less sensitive to antioxidant treatment, with only a slight decrease, more obvious after treatment with (+)-alpha-lipoic acid $125 \mu \mathrm{mol} / \mathrm{kg} / \mathrm{day}$ compared to (+/-)-alpha-lipoic acid.

Immunoreactivity for VCAM-1 in the coronary arteries (Figure 4) was more expressed compared to the other adhesion molecules ICAM-1 and PECAM-1 (Figure 5). The 

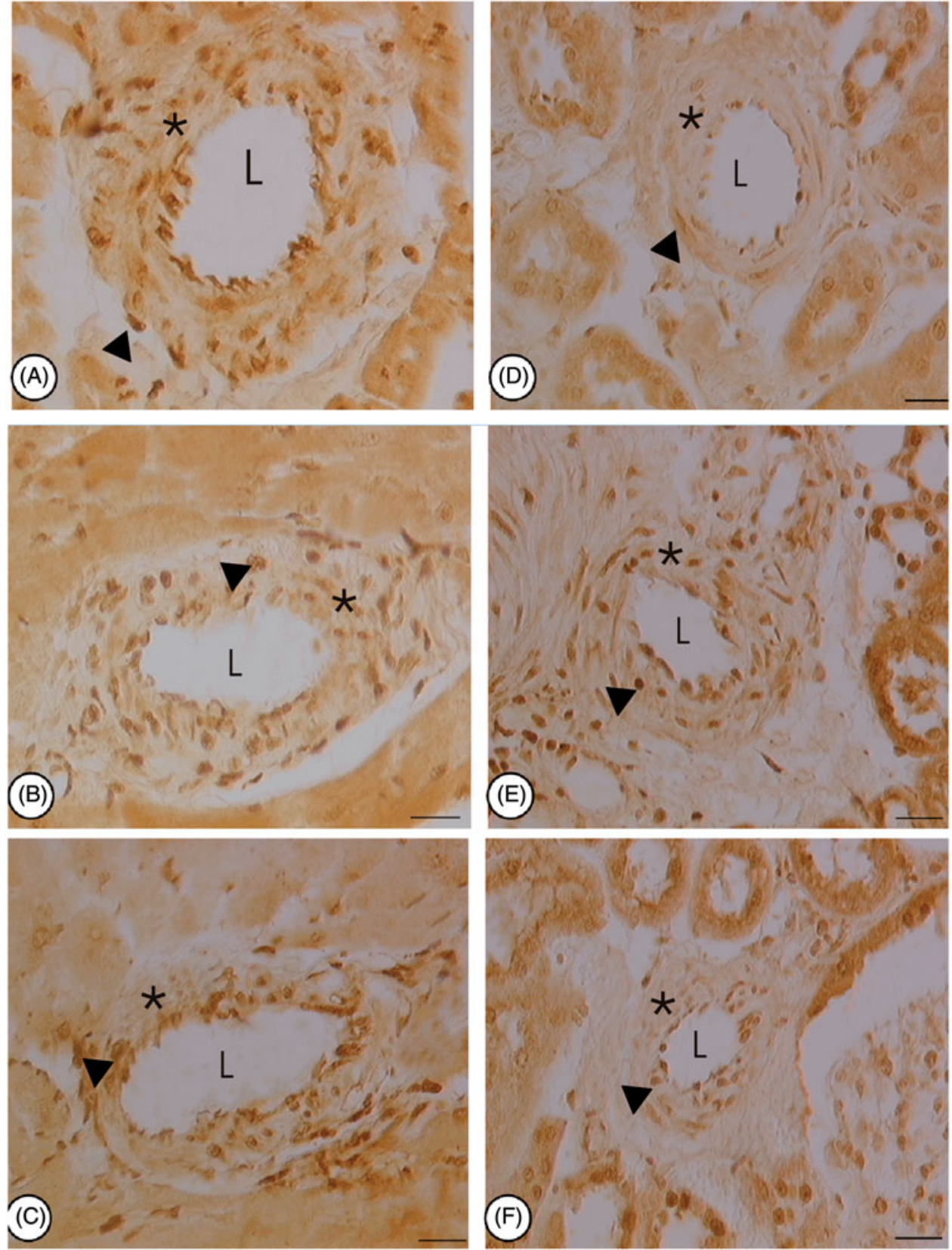

Figure 2. Sections of coronary artery branches $(A-C)$ and renal arteries (D-F) processed for the immuhistochemistry 8-oxo-dG immunohistochemistry. Arrow head: positive nucleus. Asterisks: tunica media. L: lumen. A,D: WKY + FIS B,E: SHR + FIS C,F: SHR + (+)-alpha-lipoic acid 125 $\mu$ mol/kg/ day. Calibration Bar: $25 \mu \mathrm{m}$.

immunoreaction was localized primarily at endothelial level and at the level of muscular layer of the medium-sized arteries. Immunoreaction was significantly higher in SHR compared to age matched WKY (Figures 4 and 5). The treatment with (+)-alpha-lipoic acid $125 \mu \mathrm{mol} / \mathrm{kg} / \mathrm{day}$ (Figure 4), but not with (-)-alpha-lipoic acid $125 \mu \mathrm{mol} / \mathrm{kg} /$ day countered the increase of immunoreactions for the three endothelial adhesion molecules analyzed (Figure 5).

ICAM-1, VCAM-1 and PECAM-1 expressions were significantly increased in the renal vascular endothelium of SHR (Figures 5 and 6). (+/-)-Alpha-lipoic acid $250 \mu \mathrm{mol} / \mathrm{kg} /$ day and (+)-alpha-lipoic acid $125 \mu \mathrm{mol} / \mathrm{kg} /$ day treatment prevented the rise of PECAM-1 expression on the vascular endothelium. The effect of (+)-alpha-lipoic acid $125 \mu \mathrm{mol} /$ $\mathrm{kg} /$ day (Figures 5 and 6) was more pronounced than (+/-)-alpha-lipoic acid $250 \mu \mathrm{mol} / \mathrm{kg} /$ day on PECAM-1 expression (Figure 5). ICAM-1 expression was less sensitive to antioxidant treatment, with only a slight decrease, more obvious after treatment with (+)-alpha-lipoic acid $125 \mu \mathrm{mol} /$ $\mathrm{kg} /$ day (Figures 5 and 6) compared to (+/-)-alpha-lipoic acid $250 \mu \mathrm{mol} / \mathrm{kg} /$ day (Figure 5).

\section{Discussion}

Long lasting arterial hypertension leads to vascular injury. The severity of this damage is closely related to the degree of blood pressure elevation and to the increased thickness of arterial wall. These changes increase the wall-to-lumen ratio and accordingly arterial resistance $(4,5)$.

Oxidative stress and endothelial dysfunction is consistently observed in hypertensive subjects, but emerging evidence suggests that they also have a causal role in 


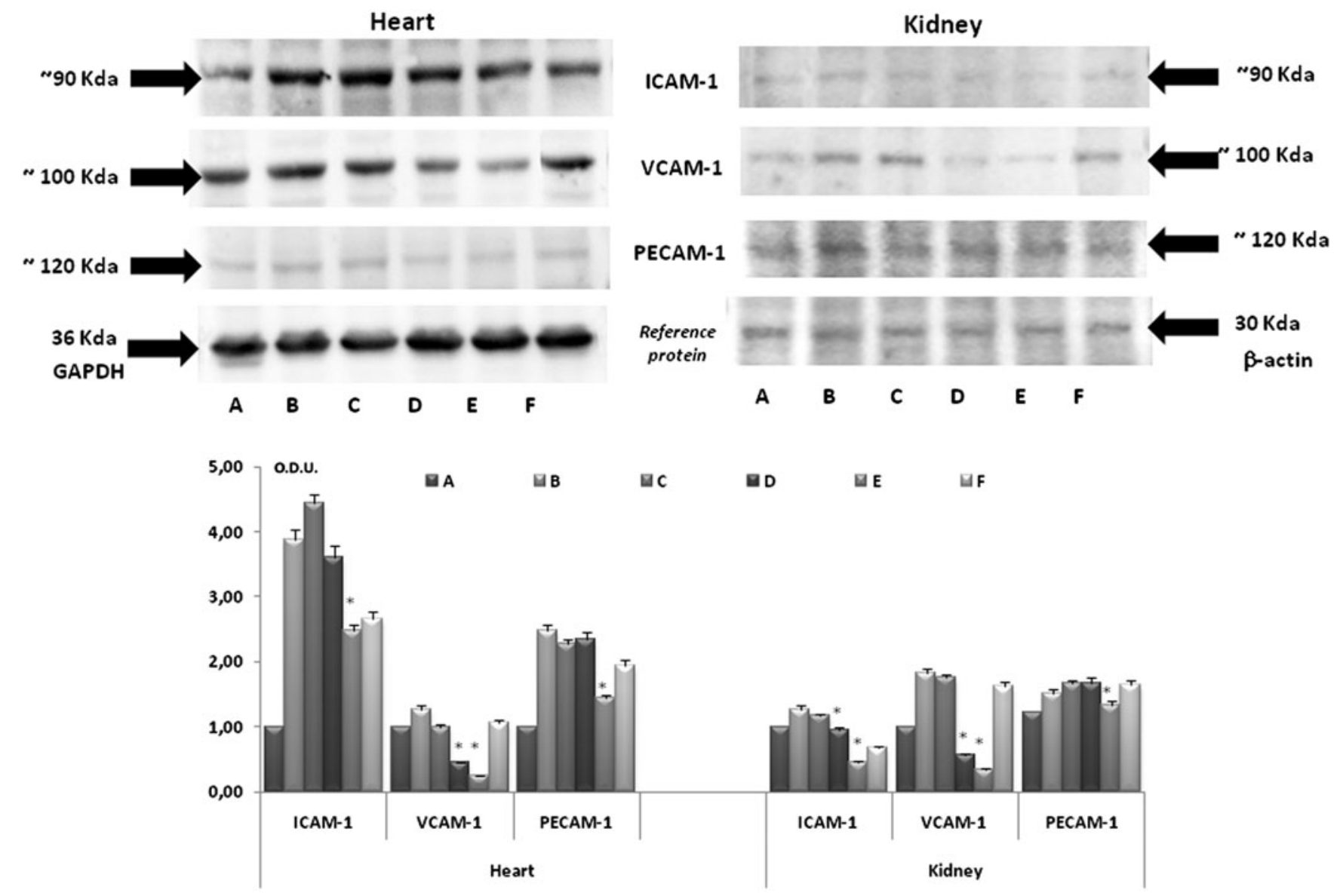

Figure 3. Immunochemical analysis of the heart and kidney processed with different antibodies. A: WKY + FIS B: SHR + FIS C: SHR + (+/-)-alpha lipoic acid $125 \mu \mathrm{mol} / \mathrm{kg} / \mathrm{day}$; D:SHR CCI + (+/-)alpha lipoic acid $250 \mu \mathrm{mol} / \mathrm{kg} / \mathrm{day} ;$ E: SHR + (+)alpha lipoic acid $125 \mu \mathrm{mol} / \mathrm{kg} / \mathrm{day} ; \mathrm{F}$ : SHR $+(-)$ alpha lipoic acid $125 \mu \mathrm{mol} / \mathrm{kg} /$ day. The densitometric data were expressed as ratio of Optical density. The results of densitometric analysis were the mean of different experiments (at least three) on the three different rats. All the values were different from WKY rats. $* p<0.05$ versus SHR + FIS rats.
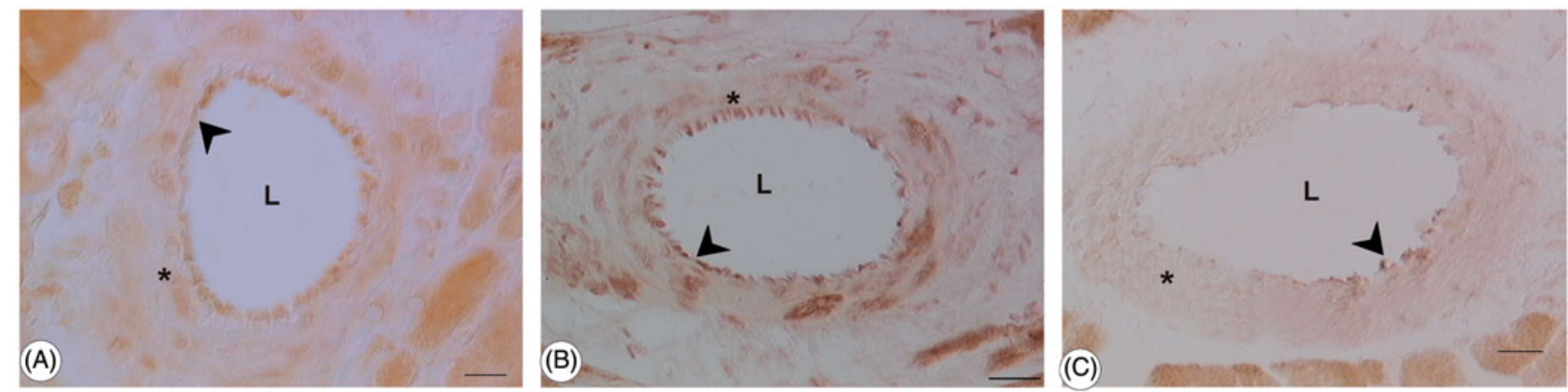

Figure 4. Sections of coronary arteries processed for VCAM-1 immunohistochemistry of. A: WKY + FIS B: SHR + FIS C: SHR + (+)alpha lipoic acid $125 \mu \mathrm{mol} / \mathrm{kg} /$ day. Note in control SHR (B) an increase expression of VCAM-1 in the endothelial layer compared to control WKY rats. Expression of VCAM-1 was lower in the SHR treated with (+)alpha lipoic acid $125 \mu \mathrm{mol} / \mathrm{kg} /$ day alpha lipoic acid. Arrow head: endothelial layer. Asterisks: tunica media. L: lumen. Calibration bar: $12,5 \mu \mathrm{m}$.

the molecular processes leading to hypertension (23). Endothelial dysfunction is defined as the imbalance between the production and bioavailability of endothelium-derived relaxing factors (EDRFs) and endothelium-derived contractile factors (EDCFs), associated with increased bioavailability of ROS and decreased antioxidant capacity characterized as oxidative stress (6).

Oxidative stress may directly alter vascular function and tone, for example, by oxidative modification of proteins or nucleic acids. ROS may directly alter vascular function or cause changes in vascular tone by several mechanisms including altered nitric oxide (NO) bioavailability or signaling. A major mechanism for the impact of oxidative stress on vascular tone is the decrease of NO bioavailability and/or signaling, leading to endothelial dysfunction, and ROS may also promote vascular cell proliferation and migration, inflammation and apoptosis, as well as extracellular matrix alterations. All of these ROS-related processes contribute to 


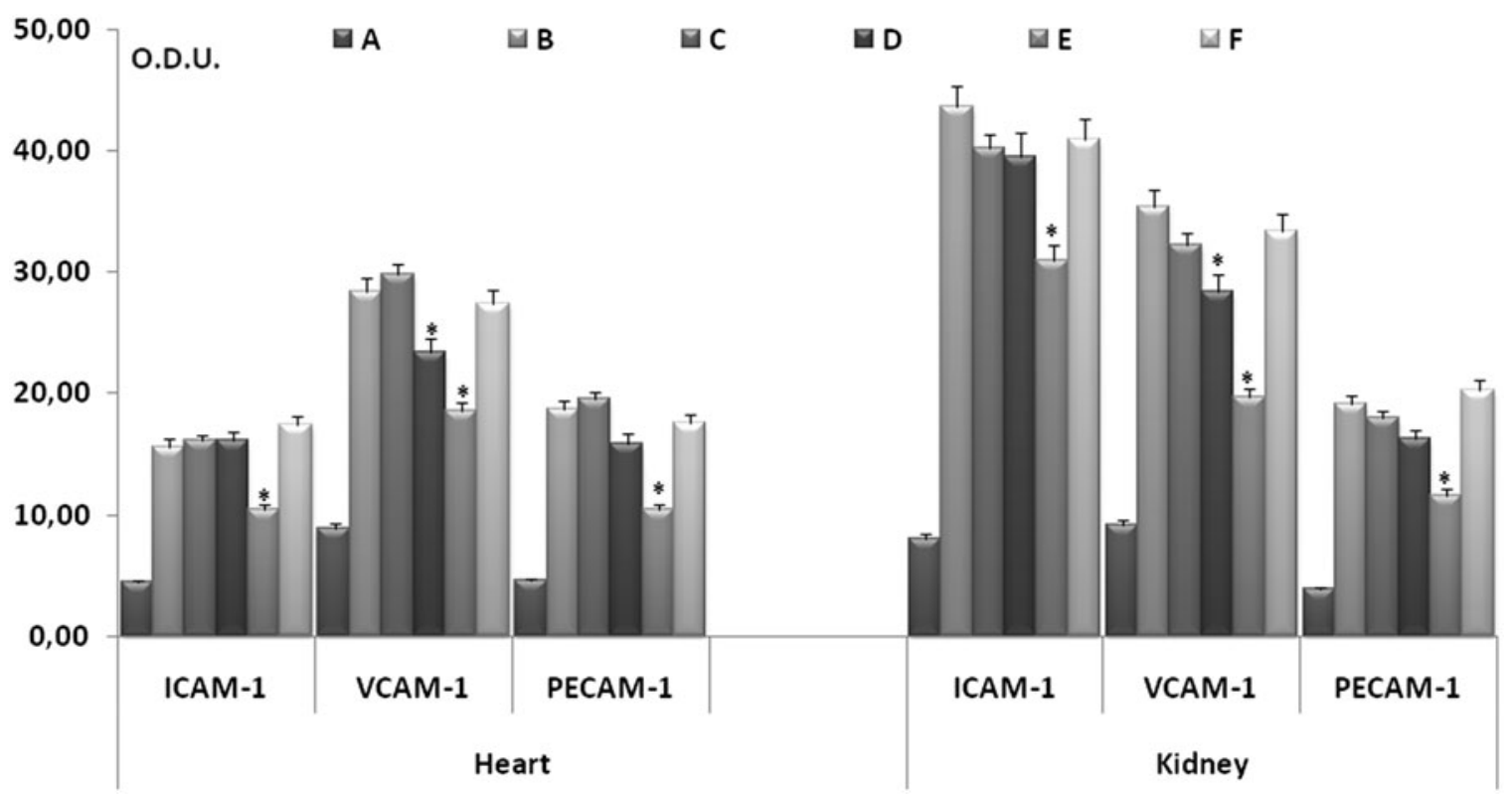

Figure 5. Densitometric analysis of immunoreaction for ICAM-1, VCAM-1 and PECAM-1 of endothelial layer of coronary and renal arteries. A: WKY + FIS B: SHR + FIS C: SHR + (+/-)-alpha lipoic acid $125 \mu \mathrm{mol} / \mathrm{kg} /$ day; D:SHR CCI + (+/-)alpha lipoic acid $250 \mu \mathrm{mol} / \mathrm{kg} / \mathrm{day} ;$ E: SHR $+(+)$ alpha lipoic acid $125 \mu \mathrm{mol} / \mathrm{kg} /$ day; F: SHR $+(-)$ alpha lipoic acid $125 \mu \mathrm{mol} / \mathrm{kg} /$ day. The densitometric data were expressed as ratio of Optical Density Unit. All the values were different from WKY rats. $* p<0.05$ versus SHR + FIS rats.

the development of hypertension (23). Reduction of vascular oxidative stress by antioxidants or ablation of ROS-producing enzymes has been shown to decrease blood pressure in animal models $(24,25)$. This evidence was in contrast to our results showing no effect of the alpha-lipoic acid treatment on blood pressure in SHR. On the other hand, alpha-lipoic acid did not modify the increase of arterial wall thickness and lumen narrowing in heart and kidney vascular tree of SHR. This is probably due to the fact that the duration of treatment was significantly different from other studies $(24,25)$ or to the different characteristics of antioxidant molecules used $(24,25)$.

However, alpha-lipoic acid countered the increase of oxidative stress, in particular in the heart. The results of present study confirm the development of oxidative stress in the heart of SHR, characterized by an increase of lipid peroxidation and nucleic acid oxidation according to other evidences regarding the increase of oxidative stress and decreased of catalase, superoxide dismutase and glutathione peroxidase activities in SHR (26). This phenomenon may be related to higher cardiac hypertrophy in SHR compared to WKY rats, indicating that the two effects are associated $(20,26)$. In contrast, renal lipid peroxidation was not apparently different between SHR and WKY rats, but nucleic acid oxidation was particularly increased in the SHR renal vessels. Although TBARS was not significantly increased in kidney of SHR, treatment with (+)-alpha-lipoic acid decreased this parameter confirming its antioxidant properties.

Endothelial cells, that represent the luminal layer of tunica intima of blood vessel, are the primary target of immunological attack in inflammatory responses. The role of vascular inflammation as an etiological factor in the progression of hypertension and hypertensive damage has been largely studied (27-29). Cyclophosphamide, an immunosuppressive agent, significantly attenuated blood pressure elevation in
SHR supporting the hypothesis regarding the involvement of inflammatory reaction in hypertension (30). The immune imbalance status correction in SHR delayed the development of hypertension (31) and therefore the immunological dysfunction is one of the etiological agent of hypertension (32). An alteration in the serum immunoglobulin levels was detected in both animals and patients, demonstrating an inflammatory involvement in hypertension (33). Furthermore, in hypertensive subjects, an increased interaction between inflammatory cells and endothelial cells (34) was demonstrated.

Inflammatory adhesion molecules, expressed by the endothelial cells, are involved in the pathogenesis of hypertension and cardiovascular events. The expression of VCAM-1 and ICAM-1 is up-regulated in response to inflammatory insult (35-38).

Hypertension also contributes to an increase in endothelial cell permeability leading to intimal edema, increases the expression of adhesion molecules $(39,40)$ and the adherence of leukocytes to the vessel wall. Therefore, hypertension directly leads to the pathological alterations of the endothelium and it seems that these effects trigger the pathogenesis of chronic vascular disease (41).

The results of the present study first confirm previous evidence concerning the wall thickness increasing and the increase of the wall to lumen ratio, in heart and kidney vasculature $(19,42,43)$. The endothelial layer of cardiac and renal arteries showed an increased expression of the ICAM-1, VCAM-1 and PECAM-1. This presence was more evident in the larger arteries than in the small one among the microvessel family (diameter $<100 \mu \mathrm{m}$ ). The adhesion molecule VCAM-1 immunoreaction was particularly high in the heart while ICAM-1 immuoreaction was higher in the kidney. The increase of endothelial adhesion molecules could be directly related to the increase of ROS production that may 

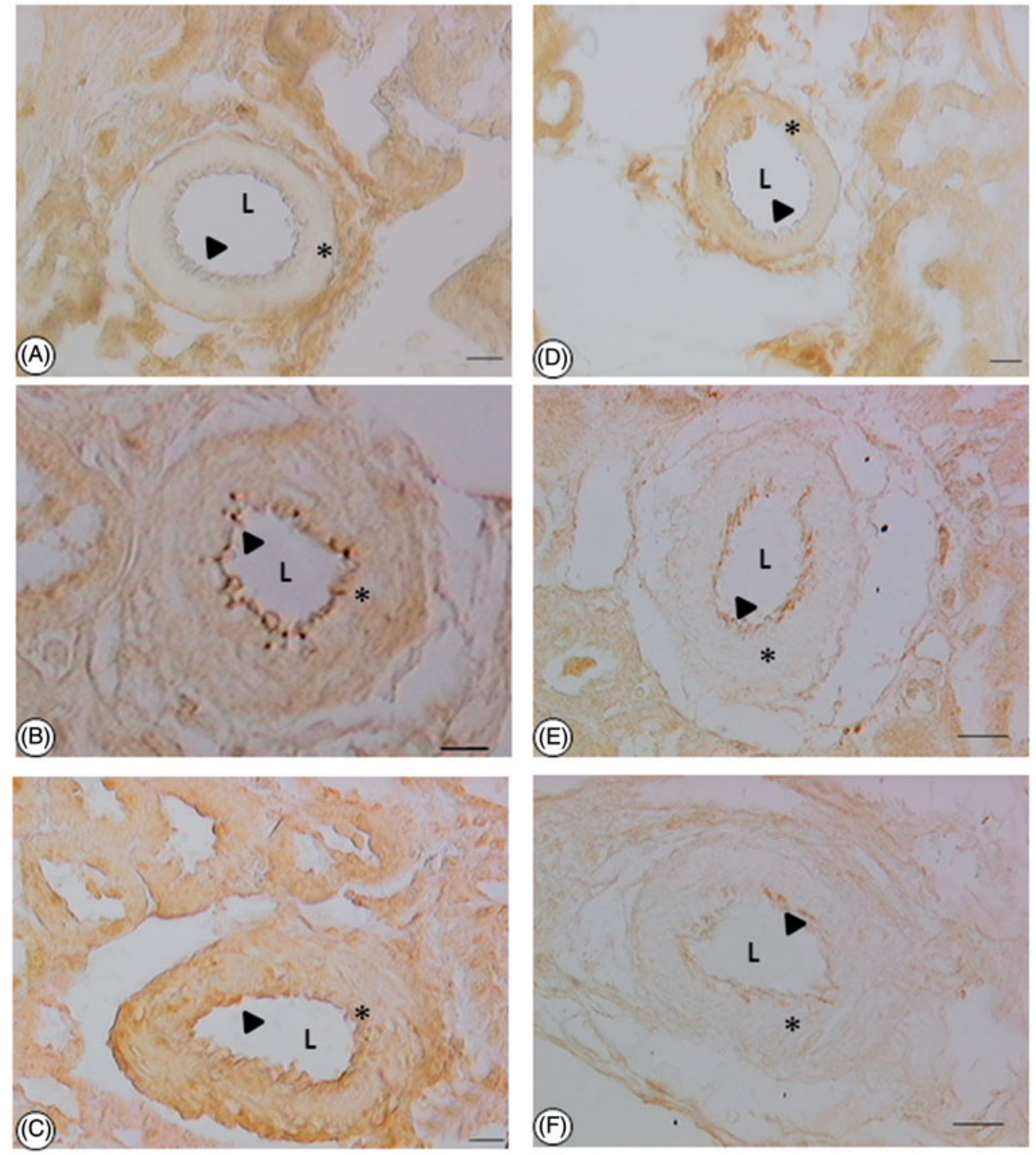

Figure 6. Sections of renal arteries processed for ICAM-1 immunohistochemistry of (A-C) and PECAM-1 (D-F). In control SHR (B-E) an increase of immunoreaction for ICAM-1 (B) and PECAM-1 (E) was noticeable compared to WKY rats. Immunoreaction was lower in rats treated with (+)alpha lipoic acid $125 \mu \mathrm{mol} / \mathrm{kg} /$ day alpha lipoic acid $(\mathrm{C}, \mathrm{F})$. Arrow: endothelial layer. Asterisks: tunica media. L: lumen. A,D: WKY + FIS; B-E: SHR + FIS; C-F: SHR + (+)-alpha lipoic acid $125 \mu \mathrm{mol} / \mathrm{kg} /$ day. Calibration bar: $25 \mu \mathrm{m}$.

also alter vascular function or cause changes in vascular tone by several mechanisms including altered NO bioavailability or signaling (23).

As a working hypothesis we can suggest that antioxidant activity of alpha-lipoic acid modify endothelial alteration in conditions of oxidative stress. In fact the expression of ICAM1, VCAM-1 and PECAM- 1 was reduced by the treatment with (+/-)-alpha-lipoic acid and to a greater extent by (+)-alphalipoic acid. This anti-inflammatory activity of alphalipoic acid can be related to its antioxidant property, by down-regulating the production of ROS. In fact, in a model of increased oxidative stress, in vitro, alpha-lipoic acid countered the ROS production $(11,44,45)$. In addition, in SHR, alpha-lipoic acid reduced the oxidative stress decreasing the plasmatic malondyaldeyde levels and oxidative status of plasmatic protein (12).

Based on these observations, the antioxidant action of alpha-lipoic acid improves the vascular functions protecting endothelial layer in cardiac and renal vascular districts. Therefore, although this antioxidant has no antihypertensive effects, its use, in particular the enantiomer $(+)$, may be beneficial in hypertensive patients to prevent inflammatory phenomena and further damage of endothelium. This antioxidant based approach, could be important in the early stage of hypertension or in its onset.

\section{Conclusion}

To sum up, it is possible to conclude that endothelial molecules investigated represent an important tool for assessing vascular injury of heart and kidney. Effects observed after treatment with (+)-alpha-lipoic acid could open new perspectives for the treatment of cardiac and renal injury of vascular nature.

\section{Declaration of interest}

The authors have no conflict of interest.

\section{References}

1. Strauer BE. Hypertensive heart disease. Berlin:Springer; 1980.

2. Mulvany MJ. Determinants of vascular characteristics haemodynamic. Hypertension 1984;6:13-25. 
3. Hall ME, do Carmo JM, da Silva AA, et al. Obesity, hypertension, and chronic kidney disease. Int J Nephrol Renovasc Dis 2014;7: $75-88$.

4. Heagerty AM, Aalkjaer C, Bund SJ, et al. Small artery structure in hypertension: dual processes of remodeling and growth. Hypertension 1993;21:391-7.

5. Baumbach GL, Heistad DD. Remodeling of cerebral arterioles in chronic hypertension. Hypertension 1989;13:968-72.

6. Silva BR, Pernomian L, Bendhack LM. Contribution of oxidative stress to endothelial dysfunction in hypertension. Front Physiol 2012;3. doi: 10.3389/fphys.2012.00441. eCollection 2012.

7. DeGraba TJ. The role of inflammation after acute stroke: utility of pursuing anti-adhesion molecule therapy. Neurology 1998;51: S62-8.

8. Emsley HC, Tyrrell PJ. Inflammation and infection in clinical stroke. J Cereb Blood Flow Metab 2002;22:1399-419.

9. Page AV, Liles WC. Biomarkers of endothelial activation/dysfunction in infectious diseases. Virulence 2013;4:507-16.

10. Bustamante J, Lodge JK, Marcocci L, et al. Alpha-lipoic acid and liver metabolism and disease. Free Radic Biol Med 1998;24: 1023-39.

11. Tomassoni D, Amenta F, Amantini C, et al. Brain activity of thioctic acid enantiomers: in vitro and in vivo studies in an animal model of cerebrovascular injury. Int J Mol Sci 2013;14: 4580-95.

12. Tomassoni D, Amenta F, Di Cesare Mannelli L, et al. Neuroprotective activity of thioctic acid in central nervous system lesions consequent to peripheral nerve injury. Biomed Res Int 2013. doi: 10.1155/2013/985093.

13. Tayebati SK, Tomassoni D, Amenta F. Spontaneously hypertensive rat as a model of vascular brain disorder: microanatomy, neurochemistry and behavior. J Neurol Sci 2012;322:241-9.

14. Sedeek M, Hébert RL, Kennedy CR, et al. Molecular mechanisms of hypertension: role of Nox family NADPH oxidases. Curr Opin Nephrol Hypertens 2009;18:122-7.

15. Amenta F, Tomassoni D. Spontaneously hypertensive rat (SHR): An animal model of vascular brain disorder. In: De Deyn PP, van Dam, D eds. Animal models of dementia. Neuromethods series. New York: Spinger; 2011;48:577-611.

16. Tomassoni D, Avola R, Mignini F, et al. Effect of treatment with choline alphoscerate on hippocampus microanatomy and glial reaction in spontaneously hypertensive rats. Brain Res 2006;1120: 183-90.

17. Sabbatini M, Catalani A, Consoli C, et al. The hippocampus in spontaneously hypertensive rats: an animal model of vascular dementia? Mech Ageing Dev 2002;123:547-59.

18. Touyz RM, Schiffrin EL. Reactive oxygen species in vascular biology: implications in hypertension. Histochem Cell Biol 2004; 122:339-52.

19. Sabbatini M, Leonardi A, Testa R, et al. Effects of dihydropyridinetype $\mathrm{Ca} 2+$ antagonists on the renal arterial tree in spontaneously hypertensive rats. J Cardiovasc Pharmacol 2002;39:39-48.

20. Amenta F, Peleg E, Tomassoni D, et al. Effect of treatment with lercanidipine on heart of Cohen-Rosenthal diabetic hypertensive rats. Hypertension 2003;41:1330-5.

21. Tayebati SK, Amenta F, Tomassoni D. Cerebrovascular and blood brain barrier morphology in spontaneously hypertensive rats: effect of treatment with choline alphoscerate. CNS Neurol Disord Drug Targets 2015;14:421-9.

22. Camussi A, Möller F, Ottaviano E, Sari Gorla M. (eds.). Metodi statistici per la sperimentazione biologica. Bologna, Italy: Zanichelli; 1986.

23. Schulz E, Gori T, Münzel T. Oxidative stress and endothelial dysfunction in hypertension. Hypertens Res 2011;34:665-73.
24. Nabha L, Garbern JC, Buller CL, Charpie JR. Vascular oxidative stress precedes high blood pressure in spontaneously hypertensive rats. Clin Exp Hypertens 2005;27:71-82.

25. Pechanova O, Zicha J, Kojsova S, et al. Effect of chronic Nacetylcysteine treatment on the development of spontaneous hypertension. Clin Sci (Lond) 2006;110:235-42.

26. Alvarez MC, Caldiz C, Fantinelli JC, et al. Is cardiac hypertrophy in spontaneously hypertensive rats the cause or the consequence of oxidative stress? Hypertens Res. 2008;31:1465-76.

27. Savoia C, Schiffrin EL. Inflammation in hypertension. Curr Opin Nephrol Hypertens 2006;15:152-8.

28. Harrison DG, Vinh A, Lob H, Madhur MS. Role of the adaptive immune system in hypertension. Curr Opin Pharmacol 2010;10: $203-7$.

29. Schiffrin EL. The immune system: role in hypertension. Can J Cardiol 2013;29:543-8

30. Khraibi AA, Norman Jr RA, Dzielak DJ. Chronic immunosuppression attenuates hypertension in Okamoto spontaneously hypertensive rats. Am J Physiol-Heart Circul Physiol 1984;16:H722-6.

31. Norman Jr RA, Dzielak DJ, Bost KL, et al. Immune system dysfunction contributes to the aetiology of spontaneous hypertension. J Hypertens 1985;3:261-8.

32. Norman Jr RA, Dzielak DJ. Spontaneous hypertension is primarily the result of sympathetic overactivity and immunologic dysfunction. Proc Soc Exp Biol Med 1986;182:448-53.

33. Dzielak DJ. The immune system and hypertension. Hypertension 1992;19:I36-44.

34. Parissis JT, Korovesis S, Giazitzoglou E, et al. Plasma profiles of peripheral monocyte-related inflammatory markers in patients with arterial hypertension: correlations with plasma endothelin-1. Int $\mathrm{J}$ Cardiol 2002;83:13-21.

35. Kim YM, Kim MY, Kim HJ, et al. Compound C independent of AMPK inhibits ICAM-1 and VCAM-1 expression in inflammatory stimulants-activated endothelial cells in vitro and in vivo. Atherosclerosis 2011;219:57-64.

36. Chiba T, Ezaki O. Dietary restriction suppresses inflammation and delays the onset of stroke in stroke-prone spontaneously hypertensive rats. Biochem Biophys Res Comm 2010;399:98-103.

37. Constans J, Conri C. Circulating markers of endothelial function in cardiovascular disease. Clin Chim Acta 2006;368:33-47.

38. Postadzhiyan AS, Tzontcheva AV, Kehayov I, Finkov B. Circulating soluble adhesion molecules ICAM-1 and VCAM-1 and their association with clinical outcome, troponin $\mathrm{T}$ and $\mathrm{C}$ reactive protein in patients with acute coronary syndromes. Clin Biochem 2008;41:126-33.

39. Suzuki K, Masawa N, Takatama M. The pathogenesis of cerebrovascular lesions in hypertensive rats. Med Electron Microsc 2001; 34:230-9.

40. Shalia KK, Mashru MR, Vasvani JB, et al. Circulating levels of cell adhesion molecules in hypertension. Indian J Clin Biochem 2009; 24:388-97.

41. Haller H. Hypertension, the endothelium and the pathogenesis of chronic vascular disease. Kidney Blood Press Res 1996;19: 166-71.

42. Sabbatini M, Vitaioli L, Baldoni E, Amenta F. Nephroprotective effect of treatment with calcium channel blockers in spontaneously hypertensive rats. J Pharmacol Exp Ther 2000; 294:948-54.

43. Sabbatini M, Strocchi P, Vitaioli L, Amenta F. Microanatomical changes of intracerebral arteries in spontaneously hypertensive rats: a model of cerebrovascular disease of the elderly. Mech Ageing Dev 2001;122:1257-68.

44. Packer L, Witt E, Tritschler HJ. Alpha-lipoic acid as a biological antioxidant. Free Radic Biol Med 1995;19:227-50.

45. Packer L, Tritschler HJ. Alpha-lipoic acid: the metabolic antioxidant. Free Radic Biol Med 1996;20:625-6. 